\title{
New Existence Results and Comparison Principles for Impulsive Integral Boundary Value Problem with Lower and Upper Solutions in Reversed Order
}

\author{
Guotao Wang, ${ }^{1}$ Lihong Zhang, ${ }^{1}$ and Guangxing Song ${ }^{2}$ \\ ${ }^{1}$ School of Mathematics and Computer Science, Shanxi Normal University, Linfen, Shanxi 041004, China \\ 2 Department of Mathematics, China University of Petroleum, Qingdao, Shandong 266555, China \\ Correspondence should be addressed to Guotao Wang, wgt2512@163.com
}

Received 29 December 2010; Accepted 3 March 2011

Academic Editor: Jin Liang

Copyright (C) 2011 Guotao Wang et al. This is an open access article distributed under the Creative Commons Attribution License, which permits unrestricted use, distribution, and reproduction in any medium, provided the original work is properly cited.

This paper investigates the existence of the extremal solutions to the integral boundary value problem for first-order impulsive functional integrodifferential equations with deviating arguments under the assumption of existing upper and lower solutions in the reversed order. The sufficient conditions for the existence of solutions were obtained by establishing several new comparison principles and using the monotone iterative technique. At last, a concrete example is presented and solved to illustrate the obtained results.

\section{Introduction}

Impulsive differential equations arise naturally from a wide variety of applications, such as control theory, physics, chemistry, population dynamics, biotechnology, industrial robotic, and optimal control ([1-4]). Therefore, it is very important to develop a general theory for differential equations with impulses including some basic aspects of this theory.

In this paper, we consider the following integral boundary value problem for firstorder impulsive functional integrodifferential equations with deviating arguments:

$$
\begin{gathered}
u^{\prime}(t)=f(t, u(t), u(\alpha(t)), W u(t), S u(t)), \quad t \in J^{\prime}, \\
\Delta u\left(t_{k}\right)=I_{k}\left(u\left(t_{k}\right)\right), \quad k=1,2, \ldots, m, \\
u(0)=r u(T)+\mu \int_{0}^{T} \omega(s, u(s)) d s+d,
\end{gathered}
$$


where $t \in J=[0, T](T>0), f \in C\left(J \times R^{4}, R\right), I_{k} \in C(R, R), \omega \in C(J \times R, R), r, \mu, d \in$ $R, \alpha \in C(J, J), 0=t_{0}<t_{1}<\cdots<t_{k}<\cdots<t_{m}<t_{m+1}=T, J^{\prime}=J \backslash\left\{t_{1}, t_{2}, \ldots, t_{m}\right\}, \Delta u\left(t_{k}\right)=$ $u\left(t_{k}^{+}\right)-u\left(t_{k}^{-}\right)$, where $u\left(t_{k}^{+}\right)$and $u\left(t_{k}^{-}\right)$denote the right and the left limits of $u(t)$ at $t=t_{k}(k=$ $1,2, \ldots, m)$, respectively, and

$$
W u(t)=\int_{0}^{\beta(t)} k(t, s) u(\gamma(s)) d s, \quad S u(t)=\int_{0}^{T} h(t, s) u(\delta(s)) d s ;
$$

here $\beta, \gamma, \delta \in C(J, J), k(t, s) \in C\left(D, R^{+}\right), h(t, s) \in C\left(J \times J, R^{+}\right), D=\left\{(t, s) \in R^{2} \mid 0 \leq s \leq\right.$ $\beta(t), t \in J\}, R^{+}=[0,+\infty)$. Let $P C(J, R)=\left\{u: J \rightarrow R \mid u(t)\right.$ is continuous at $t \neq t_{k}$, left continuous at $t=t_{k}$ and $u\left(t_{k}^{+}\right)$exists, $\left.k=1,2, \ldots, m\right\}$ and $P C^{1}(J, R)=\{u \in P C(J, E) \mid u(t)$ is continuously differentiable at $t \neq t_{k}, u^{\prime}\left(t_{k}^{+}\right)$and $u^{\prime}\left(t_{k}^{-}\right)$exist, $\left.k=1,2, \ldots, m\right\}$. Evidently, $P C(J, R)$ and $P C^{1}(J, R)$ are Banach spaces with respective norms

$$
\|u\|_{P C}=\sup _{t \in J}|u(t)|, \quad\|u\|_{P C^{1}}=\max \left\{\|u\|_{P C},\left\|u^{\prime}\right\|_{P C}\right\} .
$$

In recent years, attention has been given to integral type of boundary conditions. The interest in the study of integral boundary conditions lies in the fact that it has various applications in applied fields such as blood flow problems, chemical engineering, thermoelasticity, underground water flow, and population dynamics. For a detailed description of the integral boundary conditions, we refer the reader to some recent papers ([5-12]) and the references therein.

The method of upper and lower solutions coupled with its associated monotone iteration scheme is an interesting and powerful mechanism that offers the theoretical as well constructive existence results for nonlinear problem in a closed set, generated by the lower and upper solutions (see [9-26]). In the above-mentioned papers, main results are formulated and proved under the assumption of existing upper and lower solutions in the usual order.

However in many cases, the lower and upper solutions occur in the reversed order. This is a fundamentally different situation. In 2009, Wang et al. [27] successfully investigated boundary value problem for functional differential equations without impulses under the assumption of existing upper and lower solutions in the reversed order. In our recent work [28], the monotone iterative technique, combining with the upper and lower solutions in the reversed order, has been successfully applied to obtain the existence of the extremal solutions for a class of nonlinear first-order impulsive functional differential equations. About other existence results for the nonordered case, see ([29-33]).

Motivated by the above-mentioned works, in this paper, we study the integral boundary value problem (1.1). As far as I am concerned, no paper has considered firstorder impulsive functional integrodifferential equations with integral boundary conditions and deviating arguments (i.e., problem (1.1)) under the assumption of existing upper and lower solutions in the reverse order. This paper fills this gap in the literature.

The rest of the paper is organized as follows. In Section 2, we establish several new comparison principles, which play an important role in the proof of main results. Further, to study the nonlinear problem (1.1), we consider the associated linear problem and obtain the uniqueness of the solutions to the associated linear problem. In Section 3, the main theorems are formulated and proved. In Section 4, we give an example about integral boundary value problem for impulsive functional integrodifferential equations of mixed type (1.1). 
Advances in Difference Equations

\section{Several Comparison Principles and Linear Problem}

Lemma 2.1 (comparison result). Assume that $u \in P C^{1}(J, R)$ satisfies

$$
\begin{gathered}
u^{\prime}(t) \geq M(t) u(t)+K(t) u(\alpha(t))+N(t)(W u)(t)+L(t)(S u)(t), \quad t \in J^{\prime}, \\
\Delta u\left(t_{k}\right) \geq L_{k} u\left(t_{k}\right), \quad k=1,2, \ldots, m, \\
u(0) \geq r u(T),
\end{gathered}
$$

where $M \in C(J, R), K, N, L \in C\left(J, R^{+}\right), L_{k} \geq 0, r>0$ satisfy

(i) $r\left[1+\int_{0}^{T} q(t) d t+\sum_{0<t_{k}<T} L_{k}\right] e^{\int_{0}^{T} M(\tau) d \tau}>1$,

(ii) $\left[\int_{0}^{T} q(t) d t+\sum_{0<t_{k}<T} L_{k}\right]\left(1+r e^{\int_{0}^{T} M(\tau) d \tau}\right) \leq 1$;

here

$$
q(t)=\left[K(t) e^{\int_{0}^{\alpha(t)} M(\tau) d \tau}+N(t) \int_{0}^{\beta(t)} k(t, s) e^{\int_{0}^{\gamma(s)} M(\tau) d \tau} d s+L(t) \int_{0}^{T} h(t, s) e^{\int_{0}^{\delta(s)} M(\tau) d \tau} d s\right] e^{-\int_{0}^{t} M(\tau) d \tau}
$$

Then $u(t) \leq 0, t \in J$.

Proof. Supposing that contrary (i.e., $u(t)>0$ for some $t \in J$ ), we consider the following two possible cases:

(1) $u(t) \geq 0$ for all $t \in J$;

(2) there exist $t^{*}, t_{*} \in J$ such that $u\left(t^{*}\right)>0$ and $u\left(t_{*}\right)<0$.

$$
\begin{aligned}
& \text { Let } v(t)=u(t) e^{-\int_{0}^{t} M(\tau) d \tau} ; \text { we have } \\
& v^{\prime}(t) \geq\left[K(t) v(\alpha(t)) e^{\int_{0}^{\alpha(t)} M(\tau) d \tau}+N(t) \int_{0}^{\beta(t)} k(t, s) v(\gamma(s)) e^{\int_{0}^{\gamma(s)} M(\tau) d \tau} d s\right. \\
& \left.+L(t) \int_{0}^{T} h(t, s) v(\delta(s)) e^{\int_{0}^{\delta(s)} M(\tau) d \tau} d s\right] e^{-\int_{0}^{t} M(\tau) d \tau}, \quad t \in J^{\prime}, \\
& \Delta v\left(t_{k}\right) \geq L_{k} v\left(t_{k}\right), \quad k=1,2, \ldots, m, \\
& v(0) \geq r v(T) e^{\int_{0}^{T} M(\tau) d \tau} .
\end{aligned}
$$

Case 1. Equation (2.3) implies that $v^{\prime}(t) \geq 0$ for $t \neq t_{k}$ and $\Delta v\left(t_{k}\right) \geq 0(k=1,2, \ldots, m)$, hence, $v(t)$ is nondecreasing on $J$. By (2.3), we can get 


$$
\begin{aligned}
v^{\prime}(t) \geq & {\left[K(t) e^{\int_{0}^{\alpha(t)} M(\tau) d \tau}+N(t) \int_{0}^{\beta(t)} k(t, s) e^{\int_{0}^{\gamma(s)} M(\tau) d \tau} d s+L(t) \int_{0}^{T} h(t, s) e^{\int_{0}^{\delta(s)} M(\tau) d \tau} d s\right] } \\
& \times e^{-\int_{0}^{t} M(\tau) d \tau} v(0) .
\end{aligned}
$$

Integrating the above inequality from 0 to $t$, we have

$$
\begin{aligned}
& v(t)= v(0)+\int_{0}^{t} v^{\prime}(r) d r+\sum_{0<t_{k}<t}\left[v\left(t_{k}^{+}\right)-v\left(t_{k}\right)\right] \\
& \geq v(0)+\int_{0}^{t}\left[K(r) e^{\alpha(r)} M(\tau) d \tau+N(r) \int_{0}^{\beta(r)} k(r, s) e^{\int_{0}^{\gamma(s)} M(\tau) d \tau} d s\right. \\
&\left.+L(r) \int_{0}^{T} h(r, s) e^{\int_{0}^{\delta(s)} M(\tau) d \tau} d s\right] e^{-\int_{0}^{r} M(\tau) d \tau} v(0) d r+\sum_{0<t_{k}<t} L_{k} v\left(t_{k}\right) \\
& \geq\left\{1+\int_{0}^{t}\left[K(r) e^{\int_{0}^{\alpha(r)} M(\tau) d \tau}+N(r) \int_{0}^{\beta(r)} k(r, s) e^{\int_{0}^{\gamma(s)} M(\tau) d \tau} d s+L(r) \int_{0}^{T} h(r, s) e^{\int_{0}^{\delta(s)} M(\tau) d \tau} d s\right]\right. \\
&\left.\quad \times e^{-\int_{0}^{r} M(\tau) d \tau} d r+\sum_{0<t_{k}<t} L_{k}\right\} v(0) \\
&=\left\{1+\int_{0}^{t} q(r) d r+\sum_{0<t_{k}<t} L_{k}\right\} v(0) .
\end{aligned}
$$

Thus,

$$
v(0) \geq r v(T) e^{\int_{0}^{T} M(\tau) d \tau} \geq r\left[1+\int_{0}^{T} q(r) d r+\sum_{0<t_{k}<t} L_{k}\right] v(0) e^{\int_{0}^{T} M(\tau) d \tau}
$$

Noting condition (i), we have $v(0)=0$. Besides, $r v(T) e^{\int_{0}^{T} M(\tau) d \tau} \leq v(0)=0$, that is, $v(T) \leq 0$. Since $v(t)$ is nondecreasing on $J$, then we have $v(t) \equiv 0$, for all $t \in J$. That is, $u(t) \equiv 0$, for all $t \in J$.

Case 2. Firstly, we consider (2.3). Let $\inf _{t \in J} v(t)=-\lambda$, then $\lambda>0$, and for some $i \in\{1,2, \ldots, m\}$, there exists a $t_{*} \in\left(t_{i}, t_{i+1}\right]$, such that $v\left(t_{*}\right)=-\lambda$ or $v\left(t_{i}^{+}\right)=-\lambda$. We only consider $v\left(t_{*}\right)=-\lambda$, for the case $v\left(t_{i}^{+}\right)=-\lambda$, and the proof is similar.

By (2.3), we have

$$
\begin{aligned}
v(t) & =v(0)+\int_{0}^{t} v^{\prime}(s) d s+\sum_{0<t_{k}<t}\left[v\left(t_{k}^{+}\right)-v\left(t_{k}\right)\right] \\
& \geq v(0)-\lambda\left[\int_{0}^{t} q(s) d s+\sum_{0<t_{k}<t} L_{k}\right] .
\end{aligned}
$$


Advances in Difference Equations

Let $t=t_{*}$ in (2.7); we have

$$
-\lambda \geq v(0)-\lambda\left[\int_{0}^{t} q(s) d s+\sum_{0<t_{k}<t} L_{k}\right] .
$$

So,

$$
v(0) \leq-\lambda+\lambda\left[\int_{0}^{T} q(s) d s+\sum_{0<t_{k}<T} L_{k}\right]
$$

On the other hand,

$$
v(t)=v(T)-\int_{t}^{T} v^{\prime}(s) d s-\sum_{t \leq t_{k}<T}\left[v\left(t_{k}^{+}\right)-v\left(t_{k}\right)\right] .
$$

Let $t=t^{*}$ in $(2.10)$, then

$$
0<u\left(t^{*}\right) e^{-\int_{0}^{*^{*}} M(\tau) d \tau}=v\left(t^{*}\right)=v(T)-\int_{t^{*}}^{T} v^{\prime}(s) d s-\sum_{t^{*} \leq t_{k}<T}\left[v\left(t_{k}^{+}\right)-v\left(t_{k}\right)\right] .
$$

That is,

$$
v(T)>\int_{t^{*}}^{T} v^{\prime}(s) d s+\sum_{t^{*} \leq t_{k}<T}\left[v\left(t_{k}^{+}\right)-v\left(t_{k}\right)\right]
$$

By (2.3), we have

$$
v(T)>-\lambda\left[\int_{0}^{T} q(s) d s+\sum_{0<t_{k}<T} L_{k}\right] .
$$

Thus, by (2.9), (2.13), and $v(0) \geq r v(T) e^{\int_{0}^{T} M(\tau) d \tau}$, we obtain

$$
-\lambda\left[\int_{0}^{T} q(s) d s+\sum_{0<t_{k}<T} L_{k}\right] r e^{\int_{0}^{T} M(\tau) d \tau}<-\lambda+\lambda\left[\int_{0}^{T} q(s) d s+\sum_{0<t_{k}<T} L_{k}\right] .
$$

So, $\left[\int_{0}^{T} q(t) d t+\sum_{0<t_{k}<T} L_{k}\right]\left(1+r e^{\int_{0}^{T} M(\tau) d \tau}\right)>1$, which contradicts condition (ii). Hence, $u(t) \leq 0$ on $J$.

The proof of Lemma 2.1 is complete.

Corollary 2.2. Assume that $M \in C(J, R), K, N, L \in C\left(J, R^{+}\right), L_{k} \geq 0, r e^{\int_{0}^{T} M(\tau) d \tau}>1$, and condition (ii) in Lemma 2.1 hold. Let $u \in P C^{1}(J, R)$ satisfy (2.1). Then $u(t) \leq 0, t \in J$.

Proof. The proof of Corollary 2.2 is easy, so we omit it.

Lemma 2.3 (comparison result). Let $u \in P C^{1}(J, R)$ satisfy (2.1). Assume that $M, K, N, L \in$ $C(J,[0,+\infty)), L_{k} \geq 0, r \geq 0$ and condition (i) in Lemma 2.1 hold. In addition assume that 
(iii) $\int_{0}^{T}\left[M(t)+K(t)+N(t) \int_{0}^{\beta(t)} k(t, s) d s+L(t) \int_{0}^{T} h(t, s) d s\right] d t+\sum_{0<t_{k}<T} L_{k} \leq 1 /(r+1)$.

Then $u(t) \leq 0, t \in J$.

Proof. The proof is similar to the proof of Lemma 2.1 [28], so we omit it.

Corollary 2.4. Assume that $M, K, N, L \in C(J,[0,+\infty)), \int_{0}^{T} M(t) d t>0, L_{k} \geq 0, r \geq 1$, and condition (iii) in Lemma 2.3 hold. Let $u \in P C^{1}(J, R)$ satisfy (2.1). Then $u(t) \leq 0, t \in J$.

Proof. The proof of Corollary 2.4 is easy, so we omit it.

Remark 2.5. Corollary 2.4 holds for $r>1$ if we delete $\int_{0}^{T} M(t) d t>0$.

Remark 2.6. In the special case where (2.1) does not contain the operators $W u(t)=$ $\int_{0}^{\beta(t)} k(t, s) u(\gamma(s)) d s$ and $S u(t)=\int_{0}^{T} h(t, s) u(\delta(s)) d s$, Lemmas 2.1 and 2.3 develop Lemma 2.1 [28], and Corollaries 2.2 and 2.4 develop Corollary 2.1 [28]. Moreover, the condition $M \in C(J, R)$ in Lemma 2.1 and Corollary 2.2 is more extensive than the corresponding condition in [28], and if we let $N(t)=L(t)=0$ in Lemma 2.3 and Corollary 2.4, we can obtain Lemma 2.1 and Corollary 2.1 in [28], respectively. Therefore, our comparison results in this paper develop and generalize the corresponding results in [28].

To study the nonlinear problem (1.1), we first consider the associated linear problem

$$
\begin{gathered}
u^{\prime}(t)=\sigma(t)+M(t) u(t)+K(t) u(\alpha(t))+N(t)(W u)(t)+L(t)(S u)(t), \quad t \in J^{\prime}, \\
\Delta u\left(t_{k}\right)=\gamma_{k}+L_{k} u\left(t_{k}\right), \quad k=1,2, \ldots, m, \\
u(0)=r u(T)+b,
\end{gathered}
$$

where $\sigma \in P C(J, R), \gamma_{k}, b \in R$.

Definition 2.7. One says $u \in P C^{1}(J, R)$ is a solution of (2.15) if it satisfies (2.15).

Definition 2.8. One says that $u \in P C^{1}(J, R)$ is called a lower solution of (2.15) if

$$
\begin{gathered}
u^{\prime}(t) \leq \sigma(t)+M(t) u(t)+K(t) u(\alpha(t))+N(t)(W u)(t)+L(t)(S u)(t), \quad t \in J^{\prime}, \\
\Delta u\left(t_{k}\right) \leq \gamma_{k}+L_{k} u\left(t_{k}\right), \quad k=1,2, \ldots, m, \\
u(0) \leq r u(T)+b,
\end{gathered}
$$

and it is an upper solution of (2.15) if the above inequalities are reversed.

Lemma 2.9. Let all assumptions of Lemma 2.1 hold. In addition assume that $u_{0}, v_{0} \in P C^{1}(J, R)$ are lower and upper solutions of (2.15), respectively, and $u_{0}(t) \geq v_{0}(t)$, for all $t \in J$. Then the problem (2.15) has a unique solution $w \in P C^{1}(J, R)$.

Proof. The proof is similar to the proof of Lemma 2.2 [28], so we omit it.

Remark 2.10. In Lemma 2.9, if we replace "Lemma 2.1" by any of "Corollary 2.2", "Lemma 2.3", or "Corollary 2.4", then the conclusion of Lemma 2.9 holds. 
Advances in Difference Equations

\section{Nonlinear Problem}

Definition 3.1. One says $u \in P C^{1}(J, R)$ is a solution of (1.1) if it satisfies (1.1).

Definition 3.2. One says that $u \in P C^{1}(J, R)$ is called a lower solution of (1.1) if

$$
\begin{gathered}
u^{\prime}(t) \leq f(t, u(t), u(\alpha(t)), W u(t), S u(t)), \quad t \in J^{\prime}, \\
\Delta u\left(t_{k}\right) \leq I_{k}\left(u\left(t_{k}\right)\right), \quad k=1,2, \ldots, m, \\
u(0) \leq r u(T)+\mu \int_{0}^{T} \omega(s, u(s)) d s+d,
\end{gathered}
$$

and it is an upper solution of (1.1) if the above inequalities are reversed.

Theorem 3.3. Let all assumptions of Lemma 2.1 hold. In addition assume that

$\left(H_{1}\right) u_{0}, v_{0} \in P C^{1}(J, R)$ are lower and upper solutions of $(1.1)$, respectively, and $u_{0}(t) \geq v_{0}(t)$, for all $t \in J$;

$\left(H_{2}\right)$ the function $f \in C\left(J \times R^{4}, R\right)$ satisfies

$$
f(t, u, v, w, z)-f(t, \bar{u}, \bar{v}, \bar{w}, \bar{z}) \leq M(t)(u-\bar{u})+K(t)(v-\bar{v})+N(t)(w-\bar{w})+L(t)(z-\bar{z})
$$

for $v_{0}(t) \leq \bar{u} \leq u \leq u_{0}(t), v_{0}(\alpha(t)) \leq \bar{v} \leq v \leq u_{0}(\alpha(t)), W v_{0}(t) \leq \bar{w} \leq w \leq W u_{0}(t), S v_{0}(t) \leq$ $\bar{z} \leq z \leq S u_{0}(t)$, for all $t \in J$;

$\left(H_{3}\right)$ the function $I_{k} \in C(R, R)$ satisfies

$$
I_{k}(u)-I_{k}(\bar{u}) \leq L_{k}(u-\bar{u})
$$

for $v_{0}\left(t_{k}\right) \leq \bar{u} \leq u \leq u_{0}\left(t_{k}\right), k=1,2, \ldots, m ;$

$\left(H_{4}\right)$ there exists $a(t) \in C\left(J, R^{+}\right)$such that

$$
\mu \int_{0}^{T}[\omega(t, v)-\omega(t, \bar{v})] d t \leq-a(t)(v-\bar{v})
$$

$$
\text { if } v_{0}(t) \leq \bar{v} \leq v \leq u_{0}(t) .
$$

Then there exist monotone iterative sequences $\left\{u_{n}\right\},\left\{v_{n}\right\}$, which converge uniformly on $J$ to the extremal solutions of $(1.1)$ in $\left[v_{0}, u_{0}\right]=\left\{u \in P C^{1}(J, R): v_{0}(t) \leq u(t) \leq u_{0}(t)\right\}$.

Proof. For any $\eta \in\left[v_{0}, u_{0}\right]$, we consider the problem

$$
\begin{gathered}
u^{\prime}(t)=\sigma_{\eta}(t)+M(t) u(t)+K(t) u(\alpha(t))+N(t)(W u)(t)+L(t)(S u)(t), \quad t \in J^{\prime}, \\
\Delta u\left(t_{k}\right)=\gamma_{k}+L_{k} u\left(t_{k}\right), \quad k=1,2, \ldots, m, \\
u(0)=r u(T)+b,
\end{gathered}
$$


where

$$
\begin{aligned}
\sigma_{\eta}(t)= & f(t, \eta(t), \eta(\alpha(t)), W \eta(t), S \eta(t)) \\
& -M(t) \eta(t)-K(t) \eta(\alpha(t))-N(t)(W \eta)(t)-L(t)(S \eta)(t), \\
\gamma_{k}= & I_{k}\left(\eta\left(t_{k}\right)\right)-L_{k} \eta\left(t_{k}\right), \quad b=\mu \int_{0}^{T} \omega(s, \eta(s)) d s+d .
\end{aligned}
$$

Firstly, we verify that $u_{0}, v_{0}$ are lower and upper solutions in the reversed order of (3.5). By $\left(H_{1}\right) \sim\left(H_{4}\right)$, we obtain, for $t \neq t_{k}$,

$$
\begin{aligned}
u_{0}^{\prime}(t) \leq & f\left(t, u_{0}(t), u_{0}(\alpha(t)), W u_{0}(t), S u_{0}(t)\right) \\
\leq & f(t, \eta(t), \eta(\alpha(t)), W \eta(t), S \eta(t))-M(t) \eta(t)-K(t) \eta(\alpha(t))-N(t)(W \eta)(t) \\
& -L(t)(S \eta)(t)+M(t) u_{0}(t)+K(t) u_{0}(\alpha(t))+N(t)\left(W u_{0}\right)(t)+L(t)\left(S u_{0}\right)(t) \\
= & \sigma_{\eta}(t)+M(t) u_{0}(t)+K(t) u_{0}(\alpha(t))+N(t)\left(W u_{0}\right)(t)+L(t)\left(S u_{0}\right)(t),
\end{aligned}
$$

and, analogously,

$$
v_{0}^{\prime}(t) \geq \sigma_{\eta}(t)+M(t) v_{0}(t)+K(t) v_{0}(\alpha(t))+N(t)\left(W v_{0}\right)(t)+L(t)\left(S v_{0}\right)(t) .
$$

Besides, for $t=t_{k}$,

$$
\begin{gathered}
\Delta u_{0}\left(t_{k}\right) \leq I_{k}\left(u_{0}\left(t_{k}\right)\right) \leq I_{k}\left(\eta\left(t_{k}\right)\right)-L_{k} \eta\left(t_{k}\right)+L_{k} u_{0}\left(t_{k}\right)=\gamma_{k}+L_{k} u_{0}\left(t_{k}\right), \\
\Delta v_{0}\left(t_{k}\right) \geq \gamma_{k}+L_{k} v_{0}\left(t_{k}\right) .
\end{gathered}
$$

In addition,

$$
\begin{aligned}
u_{0}(0) \leq r u_{0}(T)+\mu \int_{0}^{T} \omega\left(s, u_{0}(s)\right) d s+d & \leq r u_{0}(T)+\mu \int_{0}^{T} \omega(s, \eta(s)) d s+d=r u_{0}(T)+b, \\
v_{0}(0) & \geq r v_{0}(T)+b .
\end{aligned}
$$

Therefore, $u_{0}, v_{0}$ are lower and upper solutions in the reversed order of (3.5). By Lemma 2.9, we know that (3.5) has a unique solution $w \in P C^{1}(J, R)$.

Now, we prove that $w \in\left[v_{0}, u_{0}\right]$. Let $p=w-u_{0}$; we can get

$$
\begin{gathered}
p^{\prime}(t) \geq M(t) p(t)+K(t) p(\alpha(t))+N(t)(W p)(t)+L(t)(S p)(t), \quad t \in J^{\prime} \\
\Delta p\left(t_{k}\right) \geq L_{k} p\left(t_{k}\right), \quad k=1,2, \ldots, m, \\
p(0) \geq r p(T) .
\end{gathered}
$$

By Lemma 2.1, we have that $p(t) \leq 0$, for all $t \in J$. That is, $w \leq u_{0}$. Similarly, we can show that $v_{0} \leq w$. Therefore, we have $w \in\left[v_{0}, u_{0}\right]$. 
Next, we denote an operator $A:\left[v_{0}, u_{0}\right] \rightarrow\left[v_{0}, u_{0}\right]$ by $u=A \eta$. We prove that $A$ is nondecreasing. Let $\eta_{1}, \eta_{2} \in\left[v_{0}, u_{0}\right]$ such that $\eta_{1} \leq \eta_{2}$. Setting $p=u_{1}-u_{2}, u_{1}=A \eta_{1}, u_{2}=A \eta_{2}$, by $\left(H_{2}\right) \sim\left(H_{4}\right)$, we have

$$
\begin{aligned}
p^{\prime}(t)= & f\left(t, \eta_{1}(t), \eta_{1}(\alpha(t)), W \eta_{1}(t), S \eta_{1}(t)\right)-M(t) \eta_{1}(t)-K(t) \eta_{1}(\alpha(t))-N(t)\left(W \eta_{1}\right)(t) \\
& -L(t)\left(S \eta_{1}\right)(t)+M(t) u_{1}(t)+K(t) u_{1}(\alpha(t))+N(t)\left(W u_{1}\right)(t)+L(t)\left(S u_{1}\right)(t) \\
& -f\left(t, \eta_{2}(t), \eta_{2}(\alpha(t)), W \eta_{2}(t), S \eta_{2}(t)\right)+M(t) \eta_{2}(t)+K(t) \eta_{2}(\alpha(t))+N(t)\left(W \eta_{2}\right)(t) \\
& +L(t)\left(S \eta_{2}\right)(t)-M(t) u_{2}(t)-K(t) u_{2}(\alpha(t))-N(t)\left(W u_{2}\right)(t)-L(t)\left(S u_{2}\right)(t) \\
\geq & M(t) p(t)+K(t) p(\alpha(t))+N(t)(W p)(t)+L(t)(S p)(t), \quad t \in J^{\prime}, \\
\Delta p\left(t_{k}\right)= & I_{k}\left(\eta_{1}\left(t_{k}\right)\right)-L_{k} \eta_{1}\left(t_{k}\right)+L_{k} u_{1}\left(t_{k}\right)-I_{k}\left(\eta_{2}\left(t_{k}\right)\right)+L_{k} \eta_{2}\left(t_{k}\right)-L_{k} u_{2}\left(t_{k}\right) \\
\geq & L_{k} p\left(t_{k}\right), \quad k=1,2, \ldots, m, \\
p(0)= & r u_{1}(T)+\mu \int_{0}^{T} \omega\left(s, \eta_{1}(s)\right) d s+d-r u_{2}(T)-\mu \int_{0}^{T} \omega\left(s, \eta_{2}(s)\right) d s-d \\
\geq & r p(T) .
\end{aligned}
$$

By Lemma 2.1, we know $p(t) \leq 0$ on $J$, that is, $A$ is nondecreasing.

Now, let $u_{n}=A u_{n-1}, v_{n}=A v_{n-1}, n=1,2, \ldots$, then we have

$$
v_{0} \leq v_{1} \leq \cdots \leq v_{n} \leq \cdots \leq u_{n} \leq \cdots \leq u_{1} \leq u_{0}, \quad n=1,2, \cdots
$$

Obviously, $u_{n}, v_{n}(n=1,2, \ldots)$ satisfy

$$
\begin{gathered}
u_{n}^{\prime}(t)=F\left(u_{n-1}(t), u_{n}(t)\right), \quad t \in J^{\prime}, \\
\Delta u_{n}\left(t_{k}\right)=I_{k}\left(u_{n-1}\left(t_{k}\right)\right)+L_{k}\left(u_{n}-u_{n-1}\right)\left(t_{k}\right), \quad k=1,2, \ldots, m, \\
u_{n}(0)=r u_{n}(T)+\mu \int_{0}^{T} \omega\left(s, u_{n-1}(s)\right) d s+d, \\
v_{n}^{\prime}(t)=F\left(v_{n-1}(t), v_{n}(t)\right), \quad t \in J^{\prime}, \\
\Delta v_{n}\left(t_{k}\right)=I_{k}\left(v_{n-1}\left(t_{k}\right)\right)+L_{k}\left(v_{n}-v_{n-1}\right)\left(t_{k}\right), \quad k=1,2, \ldots, m, \\
v_{n}(0)=r v_{n}(T)+\mu \int_{0}^{T} \omega\left(s, v_{n-1}(s)\right) d s+d,
\end{gathered}
$$

with $F$ defined by

$$
\begin{aligned}
F(x(t), y(t))= & f(t, x(t), x(\alpha(t)), W x(t), S x(t))+M(t)(y(t)-x(t))+K(t)(y(\alpha(t))-x(\alpha(t))) \\
& +N(t)((W y)(t)-(W x)(t))+L(t)((S y)(t)-(S x)(t)) .
\end{aligned}
$$


Therefore, there exist $u^{*}, v^{*}$ such that

$$
\lim _{n \rightarrow \infty} u_{n}(t)=u^{*}(t), \quad \lim _{n \rightarrow \infty} v_{n}(t)=v^{*}(t)
$$

uniformly on $J$, and the limit functions $u^{*}, v^{*}$ satisfy (1.1). Moreover, $u^{*}, v^{*} \in\left[v_{0}, u_{0}\right]$.

Finally, we prove that $u^{*}, v^{*}$ are the extremal solutions of $(1.1)$ in $\left[v_{0}, u_{0}\right]$. Let $w \in$ $\left[v_{0}, u_{0}\right]$ be any solution of (1.1), then $A w=w$. By $v_{0} \leq w \leq u_{0}$ and the properties of $A$, we have

$$
v_{n} \leq w \leq u_{n}, \quad n=1,2, \ldots
$$

Thus, taking limit in (3.17) as $n \rightarrow \infty$, we have $v^{*} \leq w \leq u^{*}$. That is, $u^{*}, v^{*}$ are the extremal solutions of $(1.1)$ in $\left[v_{0}, u_{0}\right]$.

The proof of Theorem 3.3 is complete.

Theorem 3.4. Let conditions $\left(H_{1}\right) \sim\left(H_{4}\right)$ and all assumptions of any of Corollary 2.2, Lemma 2.3, or Corollary 2.4 satisfy, then the conclusion of Theorem 3.3 hold.

Proof. The proof is similar to the proof of Theorem 3.3, so we omit it.

\section{Example}

Consider the integral boundary value problem

$$
\begin{gathered}
u^{\prime}(t)=\frac{1}{2} t^{4}\left[t+u^{2}(t)\right]-\frac{1}{300} t^{2}\left[t-u\left(t^{2}\right)\right]^{3}-\frac{1}{500} t\left[t^{3}-\int_{0}^{t} t s u\left(s^{3}\right) d s\right]^{5} \\
-\frac{1}{700} t^{2}\left[t^{2}-\int_{0}^{1} t^{2} s u(\sqrt{s}) d s\right]^{7}, \quad t \in J=[0,1], t \neq t_{1} \\
\Delta u\left(t_{1}\right)=b \sin u\left(t_{1}\right), \quad 0 \leq b \leq \frac{26}{75} \\
u(0)=\frac{3}{2} u(1)+\mu \int_{0}^{1}\left(8 s-s^{2} u^{3}(s)\right) d s+d, \quad \mu \in R^{+}, d \in R
\end{gathered}
$$

where $m=1,0<t_{1}<1, r=3 / 2, \alpha(t)=t^{2}, \beta(t)=t, \gamma(t)=t^{3}, \delta(t)=\sqrt{t}$, for all $t \in J$.

Obviously, $u_{0}=0, v_{0}=-1$ are lower and upper solutions of (4.1), respectively, and $v_{0} \leq u_{0}$.

Note that $f(t, u, v, w, z)=(1 / 2) t^{4}\left(t+u^{2}\right)-(1 / 300) t^{2}(t-v)^{3}-(1 / 500) t\left(t^{3}-w\right)^{5}-$ $(1 / 700) t^{2}\left(t^{2}-z\right)^{7}, I_{1}(u)=b \sin u$, and $\omega(t, u)=8 t-t^{2} u^{3}$.

We have

$$
f(t, u, v, w, z)-f(t, \bar{u}, \bar{v}, \bar{w}, \bar{z}) \leq \frac{1}{25} t^{2}(v-\bar{v})+\frac{4}{25} t(w-\bar{w})+\frac{16}{25} t^{14}(z-\bar{z}),
$$

where $v_{0}(t) \leq \bar{u} \leq u \leq u_{0}(t), v_{0}(\alpha(t)) \leq \bar{v} \leq v \leq u_{0}(\alpha(t)), W v_{0}(t) \leq \bar{w} \leq w \leq$ $W u_{0}(t), S v_{0}(t) \leq \bar{z} \leq z \leq S u_{0}(t)$, for all $t \in J$. 
For $M(t)=a(t)=0, L_{1}=b, K(t)=(1 / 25) t^{2}, N(t)=(4 / 25) t, L(t)=(16 / 25) t^{14}, r=$ $3 / 2$, it is easy to verify that all conditions of Theorem 3.3 hold. Therefore, by Theorem 3.3, there exist monotone iterative sequences $\left\{u_{n}\right\},\left\{v_{n}\right\}$, which converge uniformly on $J$ to the extremal solutions of $(4.1)$ in $\left[v_{0}, u_{0}\right]$.

Remark 4.1. For appropriate and suitable choices of $b, \mu, k$, and $t_{1}$, we see that problem (4.1) has a very general form. For example, we can take $b=1 / 3, \mu=100, k=-50$, and $t_{1}=2 / 3$.

\section{Conclusions}

In this paper, we have discussed the integral boundary value problem for first-order impulsive functional integrodifferential equations with deviating arguments under the assumption of existing upper and lower solutions in the reversed order. The main results (Theorems 3.3 and 3.4) are new and the following results appear as its special cases.

(i) If we take $\alpha(t)=t$ in (1.1), we obtain the first-order impulsive ordinary integrodifferential equations with integral boundary conditions.

(ii) By taking $r=1$ and $\mu=d=0$ in (1.1), our result corresponds to periodic boundary value problem for first-order impulsive functional integrodifferential equations with deviating arguments.

(iii) For $I_{k}\left(u\left(t_{k}\right)\right)=0, k=1,2, \ldots, m$, in (1.1), we get the integral boundary value problem for first-order mixed type integrodifferential equations with deviating arguments.

\section{Acknowledgments}

The aouthors would like to express their gratitude to the anonymous reviewers and editors for their valuable comments and suggestions which led to the improvement of the original paper.

\section{References}

[1] V. Lakshmikantham, D. D. BaĬnov, and P. S. Simeonov, Theory of Impulsive Differential Equations, vol. 6 of Series in Modern Applied Mathematics, World Scientific, Teaneck, NJ, USA, 1989.

[2] D. Guo, V. Lakshmikantham, and X. Z. Liu, Nonlinear Integral Equations in Abstract Spaces, vol. 373 of Mathematics and Its Applications, Kluwer Academic Publishers, Dordrecht, The Netherlands, 1996.

[3] D. D. Bainov and P. S. Simeonov, Impulsive Differential Equations: Periodic Solutions and Applications, Longman Scientific and Technical, Harlow, UK, 1993.

[4] M. Benchohra, J. Henderson, and S. Ntouyas, Impulsive Differential Equations and Inclusions, vol. 2 of Contemporary Mathematics and Its Applications, Hindawi Publishing Corporation, New York, NY, USA, 2006.

[5] B. Ahmad, A. Alsaedi, and B. S. Alghamdi, "Analytic approximation of solutions of the forced Duffing equation with integral boundary conditions," Nonlinear Analysis: Real World Applications, vol. 9, no. 4, pp. 1727-1740, 2008.

[6] J. Jiang, L. Liu, and Y. Wu, "Second-order nonlinear singular Sturm-Liouville problems with integral boundary conditions," Applied Mathematics and Computation, vol. 215, no. 4, pp. 1573-1582, 2009.

[7] X. Zhang, M. Feng, and W. Ge, "Existence result of second-order differential equations with integral boundary conditions at resonance," Journal of Mathematical Analysis and Applications, vol. 353, no. 1, pp. 311-319, 2009. 
[8] T. Jankowski, "Positive solutions for fourth-order differential equations with deviating arguments and integral boundary conditions," Nonlinear Analysis: Theory, Methods E Applications, vol. 73, no. 5, pp. 1289-1299, 2010.

[9] G. Wang, "Boundary value problems for systems of nonlinear integro-differential equations with deviating arguments," Journal of Computational and Applied Mathematics, vol. 234, no. 5, pp. 1356-1363, 2010.

[10] G. Wang, G. Song, and Lihong Zhang, "Integral boundary value problems for first order integrodifferential equations with deviating arguments," Journal of Computational and Applied Mathematics, vol. 225, no. 2, pp. 602-611, 2009.

[11] G. Wang, L. Zhang, and G. Song, "Mixed boundary value problems for second order differential equations with different deviated arguments," Journal of Applied Mathematics \& Informatics, vol. 29, no. 1-2, pp. 191-200, 2011.

[12] G. Wang, L. Zhang, and G. Song, “Integral boundary value problem for impulsive integro-differential equations in Banach spaces," Journal of Applied Mathematics and Computing. In press.

[13] G. Wang, L. Zhang, and G. Song, "Systems of first order impulsive functional differential equations with deviating arguments and nonlinear boundary conditions," Nonlinear Analysis: Theory, Methods $\mathcal{E}$ Applications, vol. 74, no. 3, pp. 974-982, 2011.

[14] D. Franco and J. J. Nieto, "First-order impulsive ordinary differential equations with anti-periodic and nonlinear boundary conditions," Nonlinear Analysis: Theory, Methods E Applications, vol. 42, no. 2, pp. 163-173, 2000.

[15] J. J. Nieto and R. Rodríguez-López, "Boundary value problems for a class of impulsive functional equations," Computers E Mathematics with Applications, vol. 55, no. 12, pp. 2715-2731, 2008.

[16] B. Ahmad and J. J. Nieto, "Existence and approximation of solutions for a class of nonlinear impulsive functional differential equations with anti-periodic boundary conditions," Nonlinear Analysis: Theory, Methods E Applications, vol. 69, no. 10, pp. 3291-3298, 2008.

[17] R. Liang and J. Shen, "Periodic boundary value problem for the first order impulsive functional differential equations," Journal of Computational and Applied Mathematics, vol. 202, no. 2, pp. 498-510, 2007.

[18] X. Yang and J. Shen, "Nonlinear boundary value problems for first order impulsive functional differential equations," Applied Mathematics and Computation, vol. 189, no. 2, pp. 1943-1952, 2007.

[19] Z. Luo and Z. Jing, "Periodic boundary value problem for first-order impulsive functional differential equations," Computers \& Mathematics with Applications, vol. 55, no. 9, pp. 2094-2107, 2008.

[20] Z. He and X. He, "Periodic boundary value problems for first order impulsive integro-differential equations of mixed type," Journal of Mathematical Analysis and Applications, vol. 296, no. 1, pp. 8-20, 2004.

[21] T. Jankowski, "Four-point boundary-value problems for differential-algebraic systems," Journal of Optimization Theory and Applications, vol. 144, no. 1, pp. 56-68, 2010.

[22] T Jankowski, "First-order impulsive ordinary differential equations with advanced arguments," Journal of Mathematical Analysis and Applications, vol. 331, no. 1, pp. 1-12, 2007.

[23] A. Dyki and T. Jankowski, "Boundary value problems for ordinary differential equations with deviated arguments," Journal of Optimization Theory and Applications, vol. 135, no. 2, pp. 257-269, 2007.

[24] J. Li and J. Shen, "Periodic boundary value problems for delay differential equations with impulses," Journal of Computational and Applied Mathematics, vol. 193, no. 2, pp. 563-573, 2006.

[25] J. Ehme, P. W. Eloe, and J. Henderson, "Upper and lower solution methods for fully nonlinear boundary value problems," Journal of Differential Equations, vol. 180, no. 1, pp. 51-64, 2002.

[26] J. J. Nieto and R. Rodríguez-López, "Remarks on periodic boundary value problems for functional differential equations," Journal of Computational and Applied Mathematics, vol. 158, no. 2, pp. 339-353, 2003.

[27] W. Wang, X. Yang, and J. Shen, "Boundary value problems involving upper and lower solutions in reverse order," Journal of Computational and Applied Mathematics, vol. 230, no. 1, pp. 1-7, 2009.

[28] G. Wang, L. Zhang, and G. Song, "Extremal solutions for the first order impulsive functional differential equations with upper and lower solutions in reversed order," Journal of Computational and Applied Mathematics, vol. 235, no. 1, pp. 325-333, 2010.

[29] A. Cabada, P. Habets, and R. L. Pouso, “Optimal existence conditions for $\phi$-Laplacian equations with upper and lower solutions in the reversed order," Journal of Differential Equations, vol. 166, no. 2, pp. 385-401, 2000. 
[30] A. Cabada and V. Otero-Espinar, "Existence and comparison results for difference $\phi$-Laplacian boundary value problems with lower and upper solutions in reverse order," Journal of Mathematical Analysis and Applications, vol. 267, no. 2, pp. 501-521, 2002.

[31] A. Cabada, M. R. Grossinho, and F. Minhós, "Extremal solutions for third-order nonlinear problems with upper and lower solutions in reversed order," Nonlinear Analysis: Theory, Methods E Applications, vol. 62, no. 6, pp. 1109-1121, 2005.

[32] D. Jiang, Y. Yang, J. Chu, and D. O'Regan, "The monotone method for Neumann functional differential equations with upper and lower solutions in the reverse order," Nonlinear Analysis: Theory, Methods E Applications, vol. 67, no. 10, pp. 2815-2828, 2007.

[33] F. Li, M. Jia, X. Liu, C. Li, and G. Li, "Existence and uniqueness of solutions of second-order threepoint boundary value problems with upper and lower solutions in the reversed order," Nonlinear Analysis: Theory, Methods \& Applications, vol. 68, no. 8, pp. 2381-2388, 2008. 